\title{
MATHEMATICAL MODELLING OF SOIL MASSIF'S DEFORMATIONS UNDER ITS DRAINAGE
}

\author{
M.T. Kuzlo ${ }^{1}$, V.S. Moshynskyi², \\ P.M. Martyniuk $3 \S$ \\ 1,2,3 National University of Water \\ and Environmental Engineering \\ Rivne, UKRAINE
}

\begin{abstract}
Mathematical models in the assessment of water saturated soil massifs' deformations under their drainage have been developed and substantiated. An algorithm for the construction of hydrodynamic networks has been developed and the transformation of differential equations of filtration and stressed-strain state of a soil mass using the method of numerical conformal mappings has been fulfilled.
\end{abstract}

AMS Subject Classification: 34B60, 35J25, 35Q74, 65M06

Key Words: filtration, stressed-deformed state, limit condition, differential equation

\section{Introduction}

The solution to this problem has started in the papers $[3,4,5,7]$, the main results of which are the development of new and improvement of the existing mathematical models and methods of their solutions for determination of the filtration flow, the stress-strain state (SSS) of the soil massifs under the action of filtration water flow. However, for the joint solution of the filtration tasks and the SSS of water-saturated ground masses under the change of the hydrogeological conditions, their further development is necessary.

Received: June 2, 2018

(c) 2018 Academic Publications

${ }^{\S}$ Correspondence author 
A mathematical model describing this problem consists of differential equations of the filtration flow $[1,2]$ and the SSS equations of the soil massif [10], which are recorded in displacements.

The paper considers the problem of full drainage of the soil massif with the known initial level of soil and groundwater surface. The final placement of groundwater is at the water resistance level.

To solve the problem, it is necessary to set the field of the filtration flow' heads, SSS of the soil massif and determine its displacement after draining at given points.

\section{Equation of filtration water flow}

Equation of filtration water flow on the primary and final time moment for two dimensional case is written in such a way

$$
\frac{\partial^{2} H}{\partial x^{2}}+\frac{\partial^{2} H}{\partial z^{2}}=0
$$

where $H(x, z)$ - water pressure in the point $(x, z)$ of soil massif, under such boundary conditions:

$$
\begin{gathered}
\frac{\partial H(x, 0)}{\partial z}=0, H(x, h(x))=h(x), \\
H(0, z)=H_{0}(z, 0), H(r, z)=H_{r}(z, 0) .
\end{gathered}
$$

The numerical solution of the boundary-value problem (1) - (3) can be obtained by the way of constructing parametric rectangular on the net in the plane $\xi, \eta$ of distinctive scheme with the usage of pattern of the type "cross" and its solution with iteration method of consequent upper relaxation.

For this reason let us look at variables $\xi, \eta$ :

$$
\xi=\xi(x, z), \eta=\eta(x, z)
$$

Under the Cauchy-Riemann equations we have:

$$
\frac{\partial \xi}{\partial x}=\frac{\partial \eta}{\partial z}, \frac{\partial \xi}{\partial z}=-\frac{\partial \eta}{\partial x} .
$$

Substituting (4) in (1), we get

$$
\frac{\partial^{2} H}{\partial \xi^{2}}\left(\frac{\partial \xi}{\partial x}\right)^{2}+\frac{\partial H}{\partial \xi} \frac{\partial^{2} \xi}{\partial x^{2}}+\frac{\partial^{2} H}{\partial \eta^{2}}\left(\frac{\partial \eta}{\partial x}\right)^{2}+\frac{\partial H}{\partial \eta} \frac{\partial^{2} \eta}{\partial x^{2}}+2 \frac{\partial^{2} H}{\partial \xi \partial \eta} \frac{\partial \xi}{\partial x} \frac{\partial \eta}{\partial x}
$$




$$
+\frac{\partial^{2} H}{\partial \xi^{2}}\left(\frac{\partial \xi}{\partial z}\right)^{2}+\frac{\partial H}{\partial \xi} \frac{\partial^{2} \xi}{\partial z^{2}}+\frac{\partial^{2} H}{\partial \eta^{2}}\left(\frac{\partial \eta}{\partial z}\right)^{2}+\frac{\partial H}{\partial \eta} \frac{\partial^{2} \eta}{\partial z^{2}}+2 \frac{\partial^{2} H}{\partial \xi \partial \eta} \frac{\partial \xi}{\partial z} \frac{\partial \eta}{\partial z}=0 .
$$

Taking into account, that $\xi(x, z), \eta(x, z)$ - interrelated harmonic functions, we have

$$
\left(\left(\frac{\partial \xi}{\partial x}\right)^{2}+\left(\frac{\partial \eta}{\partial x}\right)^{2}\right)\left(\frac{\partial^{2} H}{\partial \xi^{2}}+\frac{\partial^{2} H}{\partial \eta^{2}}\right)=0
$$

or

$$
\frac{\partial^{2} H}{\partial \xi^{2}}+\frac{\partial^{2} H}{\partial \eta^{2}}=0
$$

For the Laplace equation (5) and boundary conditions (2), (3) let us build the distinctive scheme with the usage of pattern type "cross" and solve it by the iteration method of upper relaxation:

$$
H_{i j}^{s+1}=\frac{w}{4}\left(H_{i-1, j}^{s+1}+H_{i, j-1}^{s+1}+H_{i, j+1}^{s}+H_{i+1, j}^{s}\right)+(1-w) H_{i j}^{s} .
$$

The boundary conditions of the first type mean known fixed meanings of $H$ on relative boundaries. The boundary condition of the second type in the lower boundary $\frac{\partial H(x, 0)}{\partial z}=0$ in the variables $\xi, \eta$ is the following $\frac{\partial H(x, 0)}{\partial \xi} \frac{\partial \xi}{\partial z}+$ $\frac{\partial H(x, 0)}{\partial \eta} \frac{\partial \eta}{\partial z}=0$. So far as lines of conformed net are perpendicular to the boundary, which is in such case is horizontal, then $\frac{\partial \xi}{\partial z}=-\frac{\frac{\partial x}{\partial \eta}}{J_{1}}=0$, where $J_{1}=\frac{\partial x}{\partial \xi} \frac{\partial z}{\partial \eta}-\frac{\partial x}{\partial \eta} \frac{\partial z}{\partial \xi}=\left(\frac{\partial x}{\partial \xi}\right)^{2}+\left(\frac{\partial x}{\partial \eta}\right)^{2}-$ the Jacobian of reversed mapping. So far as $\frac{\partial \eta}{\partial z} \neq 0$, the final boundary condition in the lower boundary is the following $\frac{\partial H(x, 0)}{\partial \eta}=0$. Its approximation of the first type of accuracy is the following: $H_{i 0}^{s+1}=H_{i 1}^{s+1}$.

Here we have chosen the approximation of the first order of accuracy, since only in this case the convergence of the iterative method has been proved. Iterations are carried out as long as the maximum difference between the values of the function in two consecutive iterations is greater than the given accuracy $\varepsilon$. Therefore, the stationary filtration problem has been solved numerically. In this case, the values of the pressure function have been found at all points of the conformal grid.

\section{Equation of strained deformed state of soil massif}

The mathematical model of strained deformed state of water saturated soil massif in the time moment after the stabilization of the level of soil water in 
the area lower the level of soil water $\Omega_{t}=\{(x, z) \mid x \in(0, r), z \in(0, h(x, t))\}$ is described by the following equations [8]

$$
\begin{gathered}
\mu \Delta u+(\lambda+\mu)\left(\frac{\partial^{2} u}{\partial x^{2}}+\frac{\partial^{2} w}{\partial x \partial z}\right)=\gamma_{w} \frac{\partial H}{\partial x}, \\
\mu \Delta w+(\lambda+\mu)\left(\frac{\partial^{2} u}{\partial x \partial z}+\frac{\partial^{2} w}{\partial z^{2}}\right)=\gamma_{s b}+\gamma_{w} \frac{\partial H}{\partial z},
\end{gathered}
$$

under such boundary conditions

$$
\begin{gathered}
u(x, 0)=0, w(x, 0)=0, \\
u(0, z)=0, u(r, z)=0, \\
\tau_{x z}(0, z)=0, \tau_{x z}(r, z)=0, \\
\tau_{n}\left(x, l_{1}(x)\right)=0, \sigma_{n}\left(x, l_{1}(x)\right)=\gamma_{n}\left(l_{1}(x)-l(x)\right) .
\end{gathered}
$$

Here $h(x, t)$ - the height of soil waters placement in the time moment $t ; H(x, z)$ pressure in the point $(x, z)$ in the time moment $t ; \Delta$ - the Laplace operator; $\lambda, \mu$ - resilient constants; $u(x, z)$ - horizontal and $w(x, z)$ - vertical displacements of the point, which is in the moment $t$ located in the point $(x, z) ; \gamma_{w}=\rho_{w} g$ - specific water weight ; $\gamma_{s b}$ - specific soil weight in the saturated state; $\tau_{x z}$ main tangent strains; $\sigma_{n}$-normal tensions; $\tau_{n}$ - tangent tensions; $l_{1}(x)$ - level of water level; $l(x)$ - searched for the height (depth) of soil massif in the time moment $t ; \gamma_{n}$ - specific soil weight in the natural state.

It is necessary to solve the problem that is described with (6) - (11) for the finding of soil massif's deformations from the action of filtration flow. For this reason let us write the tension across transference

$$
\tau_{x z}=\mu \varepsilon_{x z}=\frac{\mu}{2}\left(\frac{\partial u}{\partial z}+\frac{\partial w}{\partial x}\right) .
$$

In accordance with (9), $\left.u\right|_{x=0}=\left.u\right|_{x=r}=0$, that is why $\left.\frac{\partial u}{\partial z}\right|_{x=0}=\left.\frac{\partial u}{\partial z}\right|_{x=r}=$ 0 , therefore, (10) is the following:

$$
\left.\frac{\partial w}{\partial x}\right|_{x=0}=0,\left.\frac{\partial w}{\partial x}\right|_{x=r}=0 .
$$

By the way, according to there are formulae [8]:

$$
\sigma_{n}=\sigma_{x} n_{x}^{2}+\sigma_{z} n_{z}^{2}+2 \tau_{x z} n_{x} n_{z}, \tau_{n}=\sqrt{P_{x}^{2}+P_{z}^{2}-\sigma_{n}^{2}},
$$


where $P_{x}=\sigma_{x} n_{x}+\tau_{x z} n_{z}, P_{z}=\tau_{z x} n_{x}+\sigma_{z} n_{z}$ - components of tensions' vector ; $n_{x}, n_{z}$ - ditecting cosines of normal vector to the upper limit $\Omega_{1}$, that is $n_{x}=-\sin \alpha, n_{z}=\cos \alpha$, where $\alpha=\operatorname{arctg}\left(l_{1}^{\prime}(x)\right)$.

Taking into account (13) and having expressed tension across transference, from (11) we get:

$$
\begin{gathered}
\left.\left(2 \mu\left(\frac{\partial w}{\partial z}-\frac{\partial u}{\partial x}\right) n_{x} n_{z}+\frac{\mu}{2}\left(\frac{\partial u}{\partial z}+\frac{\partial w}{\partial x}\right)\left(n_{x}^{2}-n_{z}^{2}\right)\right)\right|_{z=l_{1}}=0 \\
\left(\left(\lambda\left(n_{x}^{2}+n_{z}^{2}\right)+2 \mu n_{x}^{2}\right) \frac{\partial u}{\partial x}+\left(\lambda\left(n_{x}^{2}+n_{z}^{2}\right)+2 \mu n_{z}^{2}\right) \frac{\partial w}{\partial z}\right. \\
\left.+\mu n_{x} n_{z}\left(\frac{\partial u}{\partial z}+\frac{\partial w}{\partial x}\right)\right)\left.\right|_{z=l_{1}}=\gamma_{n}\left(l_{1}(x)-l(x)\right) .
\end{gathered}
$$

The primary condition is the following:

$$
-w\left(x, l_{1}(t), t\right)=l_{0}(x)-l(x, t),
$$

where $l_{0}(x)=l(x, 0)-w\left(x, l_{1}(0), 0\right)$.

For problem solution (6) - (11), (12), (14) - (16) let us come to variables $\xi, \eta([9])$ : let us express derivative functions $\xi, \eta$ in $x, z$ through derivatives $x, z$ in $\xi, \eta$. Having substituted (4) in (6), (7), we get

$$
\begin{array}{r}
\frac{\mu}{J_{1}}\left(\frac{\partial^{2} u}{\partial \xi^{2}}+\frac{\partial^{2} u}{\partial \eta^{2}}\right)+(\lambda+\mu)\left(\frac{\partial^{2} u}{\partial \xi^{2}}\left(\frac{\partial \xi}{\partial x}\right)^{2}+2 \frac{\partial^{2} u}{\partial \xi \partial \eta} \frac{\partial \xi}{\partial x} \frac{\partial \eta}{\partial x}\right. \\
+\frac{\partial u}{\partial \xi} \frac{\partial^{2} \xi}{\partial x^{2}}+\frac{\partial^{2} u}{\partial \eta^{2}}\left(\frac{\partial \eta}{\partial x}\right)^{2}+\frac{\partial u}{\partial \eta} \frac{\partial^{2} \eta}{\partial x^{2}} \\
+\frac{\partial^{2} w}{\partial \xi^{2}} \frac{\partial \xi}{\partial x} \frac{\partial \xi}{\partial z}+\frac{\partial^{2} w}{\partial \xi \partial \eta}\left(\frac{\partial \xi}{\partial z} \frac{\partial \eta}{\partial x}+\frac{\partial \xi}{\partial x} \frac{\partial \eta}{\partial z}\right) \\
\left.+\frac{\partial^{2} w}{\partial \eta^{2}} \frac{\partial \eta}{\partial x} \frac{\partial \eta}{\partial z}+\frac{\partial w}{\partial \xi} \frac{\partial^{2} \xi}{\partial x \partial z}+\frac{\partial w}{\partial \eta} \frac{\partial^{2} \eta}{\partial x \partial z}\right)=\gamma_{w} \frac{\partial H}{\partial x} \\
\frac{\mu}{J_{1}}\left(\frac{\partial^{2} w}{\partial \xi^{2}}+\frac{\partial^{2} w}{\partial \eta^{2}}\right)+(\lambda+\mu)\left(\frac{\partial^{2} u}{\partial \xi^{2}} \frac{\partial \xi}{\partial x} \frac{\partial \xi}{\partial z}\right. \\
+\frac{\partial^{2} u}{\partial \eta^{2}} \frac{\partial \eta}{\partial x} \frac{\partial \eta}{\partial z}+\frac{\partial^{2} u}{\partial \xi \partial \eta}\left(\frac{\partial \xi}{\partial z} \frac{\partial \eta}{\partial x}+\frac{\partial \xi}{\partial x} \frac{\partial \eta}{\partial z}\right) \\
+\frac{\partial u}{\partial \xi} \frac{\partial^{2} \xi}{\partial x \partial z}+\frac{\partial u}{\partial \eta} \frac{\partial^{2} \eta}{\partial x \partial z}+\frac{\partial^{2} w}{\partial \xi^{2}}\left(\frac{\partial \xi}{\partial z}\right)^{2}+\frac{\partial^{2} w}{\partial \eta^{2}}\left(\frac{\partial \eta}{\partial z}\right)^{2}
\end{array}
$$




$$
\left.+2 \frac{\partial^{2} w}{\partial \xi \partial \eta} \frac{\partial \xi}{\partial z} \frac{\partial \eta}{\partial z}+\frac{\partial w}{\partial \xi} \frac{\partial^{2} \xi}{\partial z^{2}}+\frac{\partial w}{\partial \eta} \frac{\partial^{2} \eta}{\partial z^{2}}\right)=\gamma_{s b}+\gamma_{w} \frac{\partial H}{\partial z}
$$

The boundary conditions (8), (9) in derivatives $\xi, \eta$ are the following

$$
\begin{gathered}
u(x(\xi, \eta), 0)=0, w(x(\xi, \eta), 0)=0, \\
u(0, z(\xi, \eta))=0, u(r(\xi, \eta), z(\xi, \eta))=0,
\end{gathered}
$$

The conditions (10) on the basis of (12) $\xi, \eta$ are written in such a way:

$$
\left.\left(\frac{\partial w}{\partial \xi} \frac{\partial z}{\partial \eta}-\frac{\partial w}{\partial \eta} \frac{\partial z}{\partial \xi}\right)\right|_{\xi=0, \xi=a}=0
$$

The conditions (14), (15) variables $\xi, \eta$ are transformed analogically. Let us discrete equation (17) on the pattern of type "box"

$$
\begin{array}{r}
\frac{\mu}{J_{1}}\left(\frac{U_{i-1, j}-2 U_{i j}+U_{i+1, j}}{h_{\xi}^{2}}+\frac{U_{i, j-1}-2 U_{i j}+U_{i, j+1}}{h_{\eta}^{2}}\right) \\
+(\lambda+\mu)\left(\frac{U_{i-1, j}-2 U_{i j}+U_{i+1, j}}{h_{\xi}^{2}}\left(\frac{\partial \xi}{\partial x}\right)^{2}\right. \\
+\frac{U_{i+1, j+1}-U_{i-1, j+1}-U_{i+1, j-1}+U_{i-1, j-1}}{2 h_{\xi} h_{\eta}} \frac{\partial \xi}{\partial x} \frac{\partial \eta}{\partial x} \\
+\frac{U_{i+1, j}-U_{i-1, j}}{2 h_{\xi}} \frac{\partial^{2} \xi}{\partial x^{2}}+\frac{U_{i, j-1}-2 U_{i j}+U_{i, j+1}}{h_{\eta}^{2}}\left(\frac{\partial \eta}{\partial x}\right)^{2} \\
\left.+\frac{U_{i, j+1}-U_{i, j-1}}{2 h_{\eta}} \frac{\partial^{2} \eta}{\partial x^{2}}\right)=F_{1, i j},
\end{array}
$$

where the following symbols are used

$$
\begin{array}{r}
F_{1, i j}=\gamma_{w} \frac{\partial H}{\partial x}-(\lambda+\mu)\left(\frac{W_{i-1, j}-2 W_{i j}+W_{i+1, j}}{h_{\xi}^{2}}\right. \\
\times \frac{\partial \xi}{\partial x} \frac{\partial \xi}{\partial z}+\frac{W_{i+1, j}-W_{i-1, j}}{2 h_{\xi}} \frac{\partial^{2} \xi}{\partial x \partial z}+\frac{W_{i, j+1}-W_{i-1, j}}{2 h_{\eta}} \frac{\partial^{2} \eta}{\partial x \partial z} \\
+\frac{W_{i, j-1}-2 W_{i j}+W_{i, j+1}}{h_{\eta}^{2}} \frac{\partial \eta}{\partial x} \frac{\partial \eta}{\partial z} \\
+\frac{W_{i+1, j+1}-W_{i-1, j+1}-W_{i+1, j-1}+W_{i-1, j-1}}{2 h_{\xi} h_{\eta}}
\end{array}
$$




$$
\left.\times\left(\frac{\partial \xi}{\partial z} \frac{\partial \eta}{\partial x}+\frac{\partial \xi}{\partial x} \frac{\partial \eta}{\partial z}\right)\right)
$$

Let us accept $h_{\xi}=h_{\eta}=h$, and define the symbols

$$
\begin{gathered}
A_{1, i j}=\frac{1}{J_{1, i j} h^{2}}\left(\mu+\frac{\lambda+\mu}{J_{1, i j}}\left(\frac{z_{i j+1}-z_{i j-1}}{2 h}\right)^{2}\right), \\
B_{1, i j}=\frac{1}{J_{1, i j} h^{2}}\left(\mu+\frac{\lambda+\mu}{J_{1, i j}}\left(\frac{z_{i+1 j}-z_{i-1 j}}{2 h}\right)^{2}\right), \\
C_{1, i j}=\frac{(\lambda+\mu)}{2 h}\left(\frac{\partial^{2} \xi}{\partial x^{2}}\right)_{i j}, D_{1, i j}=\frac{(\lambda+\mu)}{2 h}\left(\frac{\partial^{2} \eta}{\partial x^{2}}\right)_{i j}, \\
E_{1, i j}=\frac{(\lambda+\mu)}{2 J_{1}^{2} h^{2}} \frac{\left(z_{i+1 j}-z_{i-1 j}\right)\left(z_{i j+1}-z_{i j-1}\right)}{4 h^{2}} .
\end{gathered}
$$

Then equation (19) will be written in the following way:

$$
\begin{array}{r}
-\left(A_{1, i j}+B_{1, i j}\right) U_{i j}+U_{i-1, j}\left(A_{1, i j}-C_{1, i j}\right)+U_{i+1, j} \\
\times\left(A_{1, i j}+C_{1, i j}\right)+U_{i, j-1}\left(B_{1, i j}-D_{1, i j}\right)+U_{i, j+1}\left(B_{1, i j}+D_{1, i j}\right) \\
-E_{1, i j}\left(U_{i+1, j+1}-U_{i-1, j+1}-U_{i+1, j-1}+U_{i-1, j-1}\right)=F_{1, i j} .
\end{array}
$$

The solution (21) will be found with the help of Gauss-Seidel method

$$
\begin{aligned}
& U_{i j}^{s+1}=\left(a_{1, i j} U_{i-1 j}^{s+1}+a_{2, i j} U_{i+1 j}^{s}+a_{3, i j} U_{i j-1}^{s+1}+a_{4, i j} U_{i j+1}^{s}\right) \\
& \quad-a_{5, i j}\left(U_{i+1 j+1}^{s}-U_{i-1 j+1}^{s+1}-U_{i+1 j-1}^{s+1}-U_{i-1 j-1}^{s+1}\right)-a_{6, i j}
\end{aligned}
$$

where

$$
\begin{gathered}
a_{1, i j}=\frac{A_{1, i j}-C_{1, i j}}{a_{0, i j}}, a_{2, i j}=\frac{A_{1, i j}+C_{1, i j}}{a_{0, i j}}, \\
a_{3, i j}=\frac{B_{1, i j}-D_{1, i j}}{a_{0, i j}}, a_{4, i j}=\frac{B_{1, i j}+D_{1, i j}}{a_{0, i j}}, \\
a_{5, i j}=\frac{E_{1, i j}}{a_{0, i j}}, a_{6, i j}=\frac{F_{1, i j}}{a_{0, i j}}, a_{0, i j}=\frac{2(\lambda+3 \mu)}{J_{1} h^{2}} .
\end{gathered}
$$

Additionally, $F_{1, i j}$ after transformation will look in the following way

$$
F_{1, i j}=\frac{\gamma_{w}}{4 h^{2} J_{1, i j}}\left(\left(H_{i+1 j}-H_{i-1 j}\right)\left(z_{i j+1}-z_{i j-1}\right)\right.
$$




$$
\begin{gathered}
\left.-\left(H_{i j+1}-H_{i j-1}\right)\left(z_{i+1 j}-z_{i-1 j}\right)\right) \\
+(\lambda+\mu)\left(\frac{W_{i-1, j}-2 W_{i j}+W_{i+1, j}}{h^{2}} \frac{\left(z_{i j+1}-z_{i j-1}\right)\left(x_{i j+1}-x_{i j-1}\right)}{4 J_{1, i j}^{2} h^{2}}\right. \\
-\frac{W_{i+1, j}-W_{i-1, j}}{2 h} \frac{\partial^{2} \xi}{\partial x \partial z}+\frac{W_{i, j+1}-W_{i, j-1}}{2 h} \frac{\partial^{2} \eta}{\partial x \partial z} \\
+\frac{W_{i, j-1}-2 W_{i j}+W_{i, j+1}}{h^{2}} \frac{\left(z_{i+1 j}-z_{i-1 j}\right)\left(x_{i+1 j}-x_{i-1 j}\right)}{4 J_{1, i j}^{2} h^{2}} \\
-\frac{W_{i+1, j+1}-W_{i-1, j+1}-W_{i+1, j-1}+W_{i-1, j-1}}{2 h^{2}} \\
\left.\times \frac{\left(x_{i j+1}-x_{i j-1}\right)\left(z_{i+1 j}-z_{i-1 j}\right)+\left(z_{i j+1}-z_{i j-1}\right)\left(x_{i+1 j}-x_{i-1 j}\right)}{4 J_{1, i j}^{2} h^{2}}\right) .
\end{gathered}
$$

Let us discrete the equation (18) on the pattern of type "box"

$$
\begin{array}{r}
\frac{\mu}{J_{1}}\left(\frac{W_{i-1, j}-2 W_{i j}+W_{i+1, j}}{h_{\xi}^{2}}+\frac{W_{i, j-1}-2 W_{i j}+W_{i, j+1}}{h_{\eta}^{2}}\right) \\
+(\lambda+\mu)\left(\frac{W_{i-1, j}-2 W_{i j}+W_{i+1, j}}{h_{\xi}^{2}}\left(\frac{\partial \xi}{\partial z}\right)^{2}\right. \\
+\frac{W_{i, j-1}-2 W_{i j}+W_{i, j+1}}{h_{\eta}^{2}}\left(\frac{\partial \eta}{\partial z}\right)^{2} \\
+\frac{W_{i+1, j+1}-W_{i-1, j+1}-W_{i+1, j-1}+W_{i-1, j-1}}{2 h_{\xi} h_{\eta}} \frac{\partial \xi}{\partial z} \frac{\partial \eta}{\partial z} \\
\left.+\frac{W_{i+1, j}-W_{i-1, j}}{2 h_{\xi}} \frac{\partial^{2} \xi}{\partial z^{2}}+\frac{W_{i, j+1}-W_{i, j-1}}{2 h_{\eta}} \frac{\partial^{2} \eta}{\partial z^{2}}\right)=F_{2},
\end{array}
$$

where

$$
\begin{gathered}
F_{2}=\gamma_{s b}+\gamma_{w} \frac{\partial H}{\partial z}-(\lambda+\mu)\left(\frac{U_{i-1, j}-2 U_{i j}+U_{i+1, j}}{h_{\xi}^{2}} \frac{\partial \xi}{\partial x} \frac{\partial \xi}{\partial z}\right. \\
+\frac{U_{i, j-1}-2 U_{i j}+U_{i, j+1}}{h_{\eta}^{2}} \frac{\partial \eta}{\partial x} \frac{\partial \eta}{\partial z}+\frac{U_{i+1, j}-U_{i-1, j}}{2 h_{\xi}} \frac{\partial^{2} \xi}{\partial x \partial z} \\
+\frac{U_{i, j+1}-U_{i, j-1}}{2 h_{\eta}} \frac{\partial^{2} \eta}{\partial x \partial z}
\end{gathered}
$$




$$
\left.+\frac{U_{i+1, j+1}-U_{i-1, j+1}-U_{i+1, j-1}+U_{i-1, j-1}}{2 h_{\xi} h_{\eta}}\left(\frac{\partial \xi}{\partial z} \frac{\partial \eta}{\partial x}+\frac{\partial \xi}{\partial x} \frac{\partial \eta}{\partial z}\right)\right) .
$$

Let us accept $h_{\xi}=h_{\eta}=h$, and define the symbols

$$
\begin{gathered}
A_{2, i j}=\frac{1}{J_{1, i j} h^{2}}\left(\mu+\frac{\lambda+\mu}{J_{1, i j}}\left(\frac{x_{i j+1}-x_{i j-1}}{2 h}\right)^{2}\right), \\
B_{2, i j}=\frac{1}{J_{1, i j} h^{2}}\left(\mu+\frac{\lambda+\mu}{J_{1, i j}}\left(\frac{x_{i+1 j}-x_{i-1 j}}{2 h}\right)^{2}\right), \\
C_{2, i j}=\frac{(\lambda+\mu)}{2 h}\left(\frac{\partial^{2} \xi}{\partial z^{2}}\right)_{i j}, D_{2, i j}=\frac{(\lambda+\mu)}{2 h}\left(\frac{\partial^{2} \eta}{\partial z^{2}}\right)_{i j}, \\
E_{2, i j}=\frac{(\lambda+\mu)}{2 J_{1}^{2} h^{2}} \frac{\left(x_{i+1 j}-x_{i-1 j}\right)\left(x_{i j+1}-x_{i j-1}\right)}{4 h^{2}} .
\end{gathered}
$$

Then equation (23) will be written in the following way:

$$
\begin{array}{r}
-\left(A_{2, i j}+B_{2, i j}\right) W_{i j}+W_{i-1, j}\left(A_{2, i j}-C_{2, i j}\right) \\
+W_{i+1, j}\left(A_{2, i j}+C_{2, i j}\right)+W_{i, j-1}\left(B_{2, i j}-D_{2, i j}\right) \\
+W_{i, j+1}\left(B_{2, i j}+D_{2, i j}\right)-E_{2, i j} \\
\times\left(W_{i+1, j+1}-W_{i-1, j+1}-W_{i+1, j-1}+W_{i-1, j-1}\right)=F_{2, i j},
\end{array}
$$

The solution (24) will be found with the help of Gauss-Seidel method

$$
\begin{aligned}
& W_{i j}^{s+1}=\left(b_{1, i j} W_{i-1 j}^{s+1}+b_{2, i j} W_{i+1 j}^{s}+b_{3, i j} W_{i j-1}^{s+1}+b_{4, i j} W_{i j+1}^{s}\right) \\
& -b_{5, i j}\left(W_{i+1 j+1}^{s}-W_{i-1 j+1}^{s+1}-W_{i+1 j-1}^{s+1}-W_{i-1 j-1}^{s+1}\right)-b_{6, i j}
\end{aligned}
$$

where

$$
\begin{gathered}
b_{1, i j}=\frac{A_{2, i j}-C_{2, i j}}{b_{0, i j}}, b_{2, i j}=\frac{A_{2, i j}+C_{2, i j}}{b_{0, i j}}, \\
b_{3, i j}=\frac{B_{2, i j}-D_{2, i j}}{b_{0, i j}}, b_{4, i j}=\frac{B_{2, i j}+D_{2, i j}}{b_{0, i j}}, \\
b_{5, i j}=\frac{E_{2, i j}}{b_{0, i j}}, \\
b_{6, i j}=\frac{F_{2, i j}}{b_{0, i j}}, b_{0, i j}=\frac{2(\lambda+3 \mu)}{J_{1, i j} h^{2}} .
\end{gathered}
$$


Additionally, $F_{2, i j}$ after transformation will be written in the following way

$$
\begin{gathered}
F_{2}=\gamma_{s b}+\frac{\gamma_{w}}{4 h^{2} J_{1, i j}}\left(-\left(H_{i+1 j}-H_{i-1 j}\right)\left(x_{i j+1}-x_{i j-1}\right)\right. \\
\left.+\left(H_{i j+1}-H_{i j-1}\right)\left(x_{i+1 j}-x_{i-1 j}\right)\right)-(\lambda+\mu) \\
\times\left(-\frac{U_{i-1, j}-2 U_{i j}+U_{i+1, j}}{h^{2}} \frac{\left(z_{i j+1}-z_{i j-1}\right)\left(x_{i j+1}-x_{i j-1}\right)}{4 J_{1, i j}^{2} h^{2}}\right. \\
\times \frac{\left(x_{i j+1}-x_{i j-1}\right)\left(z_{i+1 j}-z_{i-1 j}\right)+\left(z_{i j+1}-z_{i j-1}\right)\left(x_{i+1 j}-x_{i-1 j}\right)}{4 J_{1, i j}^{2} h^{2}} \\
+\frac{U_{i, j-1}-2 U_{i j}+U_{i, j+1}}{h^{2}} \frac{\left(z_{i+1 j}-z_{i-1 j}\right)\left(x_{i+1 j}-x_{i-1 j}\right)}{4 J_{1, i j}^{2} h^{2}} \\
\left.+\frac{U_{i+1, j}-U_{i-1, j}}{2 h} \frac{\partial^{2} \xi}{\partial x \partial z}+\frac{U_{i, j+1}-U_{i, j-1}}{2 h} \frac{\partial^{2} \eta}{\partial x \partial z}\right) .
\end{gathered}
$$

The described formulae above should be supplemented with differentiated analogue

$$
J_{1}=\left(\frac{x_{i+1 j}-x_{i-1 j}}{h}\right)^{2}+\left(\frac{x_{i j+1}-x_{i j-1}}{h}\right)^{2} .
$$

Having solved the system of linear algebraic equations (19), (23) with GaussSeidel method by formulae (22), (25), which is supplemented with the proper analogues of boundary conditions, we shall find the meanings of horizontal and vertical displacement in the given points.

The programme on the programming language $\mathrm{C}++$ has been elaborated for numerical solution of problems. Results are shown in the form of graphics in the window. There is an opportunity to save numerical results in the text file. One can look through the graphics in the any moment during the fulfillment of programme (within steps by time).

\section{Conclusions}

Vertical and horizontal displacements of water saturated soil massifs and basis under their drainage can be defined with the help of equations (22), (25) on the basis of developed and improved mathematical models of water filtration in soil environments and strained deformed soil state from the action of internal 
volumetric filtration forces. Further research can be in yielding the numerical solutions of deformed soil state in areas with variable in time curvilinear boundary and influence of soil moisture flow [6] on these displacements can be investigated.

\section{References}

[1] A.Ya. Bomba, S.V. Yaroshchak, Complex approach to modeling of twophase filtration processes under control conditions, J. of Mathematical Sciences, 184, No 1 (2012), 56-68.

[2] N. Ivanchuk, P. Martynyuk, T. Tsvetkova, O. Michuta, Mathematical modeling and computer simulation of the filtration processes in earth dams, Eastern-European J. of Enterprise Technologies, 2, No 6(86) (2017), 6369.

[3] M.H. Khulbarjan, O.O. Jushmanov, Approximate analytical solution of nonstationary filtration problem with free surface, Water Resources, 1 (1982), 107-112 (In Russian).

[4] V.S. Kremez, Modelling of soil water filtration on the basis of Boussinesq's defined equation, Hydromelioration and Hydrotechnical Construction, 31 (2006), 160-165 (In Ukrainian).

[5] M.T. Kuzlo, Modelling of deformations of natural slope under its drainage, Collected Scientific Articles (Branch Mechanical Engineering, Construction) / Polt. Nat. Techn. Univ. named after Jurija Kondratjuka, 3, No 1 (2013), 199-207 (In Ukrainian).

[6] P. Martyniuk, O. Michuta, O. Ulianchuk-Martyniuk, M. Kuzlo, Numerical investigation of pressure head jump values on a thin inclusion in one-dimensional non-linear soil moisture transport problem, International J. of Applied Mathematics, 31, No 4 (2018), 649-660; doi: 10.12732/ijam.v31i4.10; available at: http://www.diogenes.bg/ijam/index.html.

[7] P.Ja. Polubarinova-Kochina, J.M. Roger de Wiest, Theory of Ground Water Movement, Princeton University Press, Princeton (1962).

[8] I.V. Sergienko, V.V. Skopetskyi, V.S. Deineka, Mathematical Modeling and Research of Processes in Heterogeneous Environments, Naukova Dumka, Kiev (1991) (In Russian). 
[9] A.P. Vlasyuk, V.H. Myhalchuk, Automatic Building of Conformed and Quasiconformed Reflections of Fourangle Areas With the Help of Computational Mesh With "Swimming" Bonds, Preprint A USSR. Inst. Mathem., \# 89.79, Kiev (1989) (In Ukrainian).

[10] A.P. Vlasyuk, N.A. Zhukovskaya, Mathematical simulation of the stressedstrained state of the foundation of earth dams with an open surface under the influence of heat and mass transfer in the two-dimensional case, J. of Engineering Physics and Thermophysics, 88, No 2 (2015), 329-341; doi: 10.1007/s10891-015-1197-3. 